\title{
Géolinguistique
}

$18 \mid 2018$

Varia

\section{Remarques sur la « construction » de la « preuve » en géolinguistique}

Notes on "Construction" of "Proof" in Geolinguistics

\section{Anika Falkert}

\section{(2) OpenEdition}

1 Journals

Édition électronique

URL : http://journals.openedition.org/geolinguistique/315

DOI : 10.4000/geolinguistique.315

ISSN : 2650-8176

Éditeur

UGA Éditions/Université Grenoble Alpes

\section{Édition imprimée}

ISBN : 978-2-37747-073-0

ISSN : 0761-9081

\section{Référence électronique}

Anika Falkert, «Remarques sur la « construction » de la « preuve » en géolinguistique », Géolinguistique [En ligne], 18 | 2018, mis en ligne le 01 décembre 2018, consulté le 13 septembre 2019. URL : http:// journals.openedition.org/geolinguistique/315; DOI : 10.4000/geolinguistique.315

Ce document a été généré automatiquement le 13 septembre 2019.

Géolinguistique 


\title{
Remarques sur la « construction » de la « preuve » en géolinguistique
}

Notes on "Construction" of "Proof" in Geolinguistics

\author{
Anika Falkert
}

«Any representation of social reality

has to make a little out of a lot. "

(H. BECKER, Telling about Society, 2007)

\section{Introduction}

1 Howard Becker, un sociologue considéré comme l'un des héritiers de l'École de Chicago, a publié en 2017 son ouvrage le plus récent intitulé Evidence (fr. Preuve). Dans ce travail, l'auteur s'interroge sur la production des données en sociologie et sur leur interprétation en vue d'une argumentation scientifique. En partant de ses souvenirs et de ses expériences de chercheur, il nous livre une réflexion sur les conditions qui permettent la formulation d'un énoncé scientifique robuste. Ce livre fait partie d'une série de publications du même auteur portant sur la méthodologie de la recherche en sociologie, parmi lesquels on peut citer notamment Telling about Society (2007 ; trad. en fr. : Comment parler de la société, 2009) et What about Mozart? What about Murder? Reasoning from Cases (2014; trad. en fr : La bonne focale : de l'utilité des cas particuliers en sciences sociales, 2016).

2 Les réflexions épistémologiques et méthodologiques de Becker que l'on trouve dans ces trois ouvrages font suite à son étude des comportements déviants (Outsiders, 1963) et à son analyse des mondes de l'art (Art Worlds, 1982), deux recherches qui désignèrent l'auteur comme l'un des représentants de l'interactionnisme symbolique. Les défenseurs de ce courant postulent qu'une situation donnée est le résultat d'interactions entre différents agents, et que, de ce fait, il est nécessaire de prendre en compte l'ensemble des parties qui y sont impliquées afin de comprendre le fonctionnement des sociétés. Dans ce contexte, l'observation participante et l'enquête de terrain constituent le moyen privilégié du recueil de données. En reconnaissant les enjeux et les limites de cette démarche, Becker a su entamer une réflexion sur les éléments qui fondent la validité des 
démonstrations scientifiques. Or, ce travail de questionnement méthodologique et de prise de recul n'est pas l'apanage des sociologues américains. En France, la production des données sociologiques a fait l'objet d'un certain nombre de publications, dont l'article de Jean Peneff intitulé "Mesure et contrôle des observations dans un travail de terrain ", paru en 1995 dans la revue Sociétés contemporaines. Quant au rôle des données quantitatives et de l'utilisation des statistiques dans le travail d'interprétation, supposées donner une image « fidèle » de la société, on citera les travaux d'Alain Desrosières auquel Becker fait référence à plusieurs reprises.

3 Étant donné que la portée des observations de Becker dépasse largement le champ de la sociologie, il nous paraît intéressant de nous interroger, à partir des propos de cet auteur, sur la «construction" de la "preuve» en géolinguistique et de discuter quelques évolutions récentes concernant la méthodologie de recherche dans le domaine de l'étude de la variation diatopique.

\section{La triade données, preuve, idée}

4 La géolinguistique, en tant que "sous-discipline» des sciences du langage, peut être considérée comme une approche de terrain, s'appuyant massivement sur une démarche empirique basée sur des données recueillies le plus souvent par le biais d'enquêtes par questionnaire et d'entretiens. De ce fait, elle partage un certain nombre de principes méthodologiques et d'outils avec la sociologie pratiquée par Becker qui, à partir des données vérifiables, cherche à produire des idées/connaissances et valider (ou invalider) des hypothèses. L'objectif, dans les deux disciplines, peut être défini comme la « compréhension fine de phénomènes sociaux observés » (Becker, $2016: 10)$. On pourrait ajouter que, pour ce qui est de la géolinguistique, il s'agit à la fois d'un travail de documentation et d'interprétation d'un phénomène social précis qui est le comportement langagier par rapport à un espace déterminé. Dans le cadre de leur argumentation scientifique, sociologues et géolinguistes combinent habituellement trois éléments : des données, des preuves et des idées. Ces dernières constituent la base des théories ou modélisations. Selon Becker (2017:4), les données se transforment en preuves dès lors qu'elles sont utilisées pour appuyer une idée. Les trois éléments sont donc interdépendants.

5 Becker observe que les chercheurs en sciences sociales et humaines sont confrontés à des difficultés inhérentes à leur objet d'étude. Interpréter les comportements d'êtres humains équivaut à tenir compte de la complexité de leurs contextes d'apparition et à tenter de déceler progressivement les facteurs multiples qui interviennent dans la production de ces pratiques. De ce fait, l'exactitude de l'argumentation scientifique ne dépend pas forcément d'avancements sur le plan technique, c'est-à-dire d'instruments de mesure de plus en plus sophistiqués, permettant l'obtention de chiffres de plus en plus précis, mais de la capacité du chercheur à établir, à partir des données, le lien le plus juste entre données, preuves et idées.

6 En partant du constat que les choses sont toujours plus compliquées que l'on ne l'aurait cru initialement, Becker (2017:67) explique que la démarche scientifique exige de s'interroger, à chaque étape, sur les liens entre données et arguments, et de vérifier ainsi l'exactitude des connexions logiques établies par le chercheur. Le scientifique procède ainsi par tâtonnements, par approximations successives qui, parfois grâce à de nouvelles données, peuvent le conduire à la reformulation d'idées. C'est cette mise en perspective 
continue qui permet d'affiner l'interprétation des résultats et, in fine, d'apporter des réponses (parfois seulement partielles) aux questions de recherche.

7 Si un certain nombre de publications dans le domaine de la linguistique empirique se sont intéressées, pendant ces dernières décennies qui ont vu l'essor de la recherche à base de corpus, à la procédure de recueil de données, ce lien entre données, preuves et idées n'est généralement pas abordé. La plupart des manuels et « guides de bonnes pratiques » (par exemple Vaux \& Cooper, 2005; Baude \& Blanche-Benveniste, 2006; Bowern, 2008), destinés à former les (jeunes) chercheurs à la méthodologique du travail de terrain, fournissent principalement des "recettes" à appliquer. En présentant un cadre standardisé pour les recherches, ces guides ignorent délibérément de thématiser les difficultés qui peuvent empêcher les méthodes recommandées par les auteurs de fonctionner. Ainsi, les échecs possibles risquent d'être traités comme des obstacles que l'on peut surmonter en travaillant davantage et plus rigoureusement. Ceci pourrait amener à croire qu'un recueil de données selon un protocole standardisé, rigoureusement établi et validé par la communauté scientifique, garantit l'obtention de données fiables, authentiques et représentatives. Or, comme nous le verrons dans le chapitre suivant, ce n'est pas toujours le cas.

\section{Au cœur des données : à la recherche de la " représentativité » et de "l'authenticité »}

8 La sociologue et le géolinguiste partagent un certain nombre d'outils servant à la production des données, à la construction des observables. On pensera notamment aux enquêtes par questionnaire. En sciences du langage, la constitution d'importantes bases de données sur le français parlé (dont ESLO, CID, CLAPI, PFC, VALIBEL et VARILING ${ }^{1}$, pour n'en citer que quelques-unes) surtout pendant ces vingt dernières années, a considérablement modifié l'approche des variétés de français, permettant désormais aux chercheurs de s'appuyer sur des quantités importantes de données. Si l'on peut saluer le travail effectué par des équipes pour rendre accessibles ces corpus aux acteurs du monde de la recherche, il convient de s'interroger sur leurs caractéristiques et leurs limites. On sait qu'un corpus peut servir à montrer qu'un phénomène est attesté dans le parler étudié. En revanche, l'absence de certaines formes dans un corpus oral ne permet pas d'affirmer avec certitude que ces formes n'existent pas. D'autres méthodes d'investigation comme les tests d'acceptabilité s'avèrent souvent nécessaires pour enrichir les informations fournies par les corpus oraux. Les lectures de listes de mots et d'extraits de texte, employées pour documenter la variation au niveau de la prononciation, sont souvent complétées par des entretiens semi- ou non dirigés. Considéré comme un moyen économique et facile à mettre en place pour obtenir des données de production orale, l'entretien présente cependant des limites. Les inconvénients de l'entretien comme technique de recueil de la parole "naturelle", "spontanée ", ont été soulignés à maintes reprises, entre autres par Cukor-Avila et Bailey:

Even the most sophisticated [...] interviews are only surrogates for everyday, unmonitored linguistic interactions among community members: they are recreations of linguistic events, not the naturally occurring events themselves. (1995: 159)

Il s'agit donc de données provoquées, construites. Les corpus basés sur des entretiens semi-directifs sont nombreux, alors que le poids relatif de ce genre discursif (par rapport 
à d'autres formes d'interaction) est relativement faible dans la pratique du locuteur. Par ailleurs, il serait illusoire de croire que tous les entretiens sont équivalents. La relation entre enquêteur et enquêté joue un rôle déterminant dans la mesure où le fait de partager ou non des connaissances et/ou un réseau peut conduire à des produits langagiers différents, sans même parler du contenu. Ne pas tenir compte des caractéristiques de l'enquêteur équivaut à oublier qu'une interaction se construit par l'action conjointe d'au moins deux participants et que les données ainsi recueillies reflètent des processus complexes d'imitation et d'adaptation: "Chacun s'adapte sans cesse au cadre de l'interaction et aux caractéristiques de son interlocuteur. Pourquoi en serait-il autrement en enquête ? (Beaud \& Weber, $2017: 176$ et suiv.)

Les entretiens ne représentent pas un genre homogène, mais un continuum de productions qui va d'une interview en contexte formel à un type d'interaction qui ressemble à une conversation «ordinaire». Par ailleurs, il n'est pas rare que les entretiens comportent eux-mêmes plusieurs genres discursifs, ce qui peut s'avérer gênant dans le cadre d'un travail sur la grammaire d'un vernaculaire. Ainsi, un informateur qui est interrogé sur un sujet peut réorienter l'interaction vers un récit d'événements ou un récit de vie, ce qui n'est pas sans impact sur l'emploi des temps verbaux et la structure des énoncés. Faudrait-il alors réagencer ces données et rassembler des sous-corpus qui ne réunissent qu'un genre discursif afin de disposer de corpus comparables et d'éviter d'arriver à des résultats biaisés (dans le cadre d'une analyse quantitative notamment, où la sous- et la surreprésentation de certains phénomènes linguistiques s'avèrent problématique)?

11 Les débats en cours dont témoignent un certain nombre de publications (comme Scheer, 2013 et Gadet \& Wachs, 2015) montrent que le défi dans les années à venir ne peut pas être de constituer des bases de données toujours plus importantes, mais de revisiter le positionnement épistémologique, en tenant compte des besoins en matière de recueils comparables. Des initiatives récentes comme le projet CIEL-F² (v. Gadet et al., 2012), conçu comme un corpus « écologique » qui se propose de documenter, pour différentes régions de la francophonie, plusieurs genres discursifs à partir d'activités interactionnelles qui ne sont pas provoquées par le chercheur, constituent un premier pas dans cette direction.

12 La difficulté d'obtenir des données "fiables» est également liée au choix des informateurs qui sont souvent sélectionnés selon des catégories préétablies (tranches d'âge, sexe, profession, lieu de résidence et de naissance) qui sont ensuite utilisées dans le cadre de l'interprétation des données pour tirer des conclusions sur le comportement langagier des personnes appartenant aux catégories respectives. Cappeau et Gadet critiquent, à juste titre, cette démarche :

C'est faire l'hypothèse qu'un locuteur puisse être dit représentatif de quelque chose. Mais représentatif de quel aspect de son identité ? Pourquoi davantage des « hommes », des « catholiques» ou des " avocats », que des «joueurs d'échecs»? Aucun individu n'est catégorisable à partir de ses énonciations de façon rapportable à une seule dimension identitaire. (2007: 104)

13 Toujours selon Cappeau et Gadet, « on privilégie ainsi une conception du social reposant sur des catégories préconstruites, dont les locuteurs sont censés être porteurs » (ibid.).

14 S'il ne s'agit pas de rejeter des protocoles basés sur cette méthode, il nous paraît important que le chercheur soit conscient des raisonnements induits par un tel découpage, et qu'il en tienne compte dans son argumentation. 

concept : celui de l'authenticité. Dans le cadre de la documentation d'un vernaculaire, le chercheur a le souci de recueillir des données «authentiques». Mais qu'est-ce qui caractérise l'oralité «authentique»? Selon Detey (2017), l'«authenticité » d'une production donnée est en réalité constamment soumise à évaluation, et donc liée aux conditions de réception. Pour évaluer si un fait langagier, une prise de parole peuvent être considérés comme authentiques, il faudrait alors se demander pour qui, par rapport à quel usage, et dans quel contexte. Par ailleurs, ce qui est « authentique » aux yeux du chercheur ne l'est pas forcément pour l'informateur. Dans le cadre des études sur la perception des accents, la confrontation des points de vue du linguiste et du nonspécialiste est souvent indispensable afin de garantir le choix des stimuli les plus « authentiques » et "représentatifs» d'un accent. Sans remettre en question ces deux concepts, il est nécessaire d'insister sur leur caractère flou, qui impose une certaine prudence dans l'argumentation scientifique.

Outre ces difficultés, certains modes d'investigation comportent des limites évidentes dont le chercheur devrait tenir compte. Becker, en tant que sociologue ayant eu largement recours à l'enquête par questionnaire et à l'observation participante, s'interroge notamment sur l'exactitude, la justesse des réponses obtenues de cette manière et, a fortiori, sur la possibilité de les utiliser comme "preuves» dans le cadre d'une argumentation scientifique. En d'autres termes, à partir de quel moment peut-on considérer qu'une donnée est « suffisamment bonne » pour pouvoir servir de preuve?

Becker a pu observer un décalage entre les comportements déclarés et les comportements réels de ses informateurs. Plutôt que d'y voir un échec de l'enquête, il plaide pour envisager à partir de ces informations contradictoires une nouvelle problématique de recherche (v. Becker, 2017 : 14). De même, toute donnée inattendue, qui va à l'encontre des attentes du chercheur, permet de soulever de nouvelles questions, et mérite d'être mise en valeur, car elle peut annoncer des tendances et évolutions futures. C'est finalement cette démarche d'aborder le sujet sous différents angles, en fonction des données recueillies, qui, selon Becker, rend possible la production de connaissances.

Après ces remarques préliminaires qui nous ont permis de rappeler quelques difficultés rencontrées par des sociologues et des géolinguistes dans leur recherche, nous nous proposons d'aborder une problématique qui continue à recevoir beaucoup d'attention dans le domaine de la géolinguistique (l'étude des accents régionaux francophones) pour examiner plus en détail la construction de la " preuve » scientifique.

\section{4. «Construire » la «preuve »: le cas des variétés de français}

Le terme "construction" fait référence à la démarche consciente, volontaire, du chercheur de fournir des éléments qui appuient son argumentation, mais aussi au fait que les données qui peuvent être transformées en "preuves » sont généralement construites. Recueillies par le biais d'entretiens, de questionnaires, de lecture de listes de mots ou d'extraits de texte, il ne s'agit pas de matériel tout-venant, de prises de parole 
«spontanées». La pratique de la sollicitation comporte des limites par rapport à la fiabilité des «preuves » ainsi recueillies :

1. Il n'est pas toujours possible de provoquer des situations pour tous les phénomènes que l'on cherche à étudier. Que faire alors des données négatives?

2. On peut se poser la question des enseignements qui peuvent être tirés de telles productions. Le chercheur ne risque-t-il pas de tomber dans le piège d'une approche circulaire, où des données ne sont réunies que pour conforter les hypothèses qui sont à l'origine de la constitution d'un corpus?

3. Une telle approche peut conduire à n'accorder de place qu'au prévisible et limite les chances de voir surgir des catégories et des connexions non prévues à l'avance (v. Cappeau \& Gadet, $2007: 106)$.

20 Ainsi, la prudence est de mise, car faute de données complémentaires et d'une diversification des sources, il sera difficile d'affirmer que «l'étude montre que... » ou que «l'analyse des données fait apparaître que... », comme on peut le lire (trop) souvent dans les travaux qui ne s'interrogent pas forcément sur ce que les données nous disent (ou ne nous disent pas !) sur le sujet.

21 Par ailleurs, on peut se demander dans quelle mesure les données ainsi recueillies sont "objectives", une question qui n'est pas nouvelle dans la discussion linguistique, notamment en géolinguistique où elle est apparue dans le cadre des débats sur la délimitation des frontières linguistiques. Les dialectologues tentent, depuis la fin du XIX siècle, de mettre en évidence le lien entre pratiques langagières et espace, notamment à travers des isoglosses qui permettent d'illustrer / de visualiser, pour chaque phénomène, les frontières (souvent floues) qui délimitent les aires dialectales. Les travaux en dialectométrie (v. notamment Séguy, 1971 ; Guiter, 1973 ; Goebl, 1982 ; Heeringa, 2004) ont permis de mesurer la proximité (ressemblance, similarité) ou bien la distance (dissimilarité) de plusieurs ensembles de données (provenant généralement des atlas linguistiques), et de mettre en relief des structures à l'intérieur des domaines dialectaux. Cependant, la question des frontières n'est pas pour autant résolue, dans la mesure où une telle démarche ne peut témoigner «de la totalité des faits de langue, à travers pratiques, attitudes et représentations » (Viaut, 2004:7). Dans cette optique, une frontière linguistique peut être considérée comme le produit de différents acteurs sociaux qui, à travers leurs pratiques et leurs jugements, contribuent à son maintien ou à son évolution. Dans un souci de clarification, on peut être tenté de distinguer entre frontières «objectives » (établies par le chercheur) et frontières « subjectives » (définies par le locuteur naiif). Or, dans quelle mesure peut-on parler de frontières objectives?

Dans un article intitulé « What Is a Linguistic Fact?» (2001 [1975]), William Labov, en se positionnant en faveur d'une recherche basée sur une démarche empirique, remet en question le rôle de l'intuition du chercheur et s'interroge sur la nature des " preuves » dans l'élaboration de théories linguistiques. On pourrait invoquer que la linguistique de corpus permet de lever le doute sur les intuitions, car les données empiriques, "objectives", constituent des "preuves", permettant de valider ou d'infirmer les hypothèses du chercheur. Or, dans quelle mesure les données recueillies sur le terrain (dont les données concernant la variation diatopique) peuvent-elles être considérées comme objectives? Après tout, un fait linguistique n'existe qu'à partir du moment où le linguiste le repère et décide de l'étiqueter comme tel. Le problème de ce que l'on pourrait appeler la "perception sélective» du chercheur a été soulevé, entre autres, par E. Thomas, pour la transcription des faits phonétiques : 
Old, well-known variants are differentiated extensively in the transcriptions, while newer, lesser-known variants tend to be undertranscribed or ignored altogether. Perception experiments could examine the extent to which different transcribers are affected by preconceptions and by the influence of their own native dialects. $(2002: 136)$ réalité de celui qui l'a construit et du dessein qui a présidé à son élaboration (v. Scheer, 2004). Cela ne remet évidemment pas en cause l'utilité des corpus dans la recherche scientifique, mais ces réserves nous obligent à reconsidérer et à relativiser l'importance accordée aux données «objectives " par rapport à d'autres types de données. On peut alors se poser la question de savoir par quelle autre entrée (qui serait complémentaire aux données de corpus de production orale) il est possible d'aborder la question de frontières linguistiques. imposée dans les débats des dialectologues dès les années 1970. Il me parait utile de reproduire ici une discussion qui a suivi une communication présentée par Jean Séguy lors du colloque de Strasbourg en 1971 et qui a été publiée dans les actes de cette manifestation scientifique. Dans sa contribution intitulée «La fonction minimale du dialecte ", Séguy (1973) affirme que la fonction des dialectes est double : ils assurent la communication linguistique entre des groupes humains en contact et permettent à ces groupes de se différencier. Cependant, la deuxième fonction est «très humblement subordonnée à la première » $(1973: 35)$. Séguy conclut qu'il « faudrait renoncer à ce jeu lugubre et vain consistant à rechercher sur le terrain et à porter sur une carte des "limites dialectales" » (ibid.), en citant les cas du béarnais et de l'armagnacais d'un côté et du poitevin et de l'angevin de l'autre. Cette affirmation a donné lieu à un échange entre Jean Séguy, Charles Rostaing et Kurt Baldinger sur la question des frontières subjectives et objectives que je me permets de reproduire ici :

M. ROSTAING : Il n'en est pas moins vrai que, dans la conscience linguistique de ceux qui parlent, il y a un groupe de faits linguistiques qui constituent ce qu'ils appellent (à tort ou à raison) le béarnais et l'armagnacais. Je veux bien que les limites soient floues [...], mais il n'en est pas moins vrai que là, il y a un ensemble cohérent, ici un autre ensemble cohérent. Il y a des limites.

M. SÉGUY : Voyez-vous, ce sont deux points de vue tout à fait différents, et qui sont également respectables. C'est le point de vue du savant, qui compte effectivement tous les traits lexicaux, phonétiques, phonologiques, etc., et qui voit qu'il n'y a pas de limites. Et il y a le point de vue de l'usager, qu'il faut citer comme vous le faites, qui a conscience de parler béarnais et de ne pas parler armagnacais. C'est au point de vue du savant que je me plaçais. D'ailleurs, il y a parfois des concordances étonnantes, les deux points de vue peuvent concorder. Tout est possible en linguistique.

M. BALDINGER: [...] La situation me rappelle celle que nous trouvons en sémantique. Prenons deux notions comme "jour» et "nuit»; vous avez une opposition nette de sèmes, ce serait l'opposition entre le béarnais et l'armagnacais, et on peut caractériser les deux par opposition; mais dès que vous entrez dans la réalité et que vous cherchez la limite entre le jour et la nuit, il n'y en a pas. Mais il y a deux systèmes qui s'opposent. Je crois donc que les deux points de vue se complètent. (Séguy, $1973: 38$ )

L'idée d'une complémentarité de points de vue du chercheur et du locteur naïf n'est donc pas récente. Cependant, l'intégration de la linguistique populaire à la discussion linguistique n'a rien d'évident puisqu'elle semble, à première vue, en contradiction avec 
la conception de la science qui se construit sur l'objectivité, sur le quantifiable, le mesurable.

En renouant avec les outils de la dialectologie traditionnelle, la dialectologie perceptuelle (ou perceptive) tente, depuis plusieurs décennies, de cartographier les frontières subjectives que les utilisateurs établissent entre leurs propres pratiques et celle des " autres ", et apporte ainsi un éclairage nouveau sur la relation entre usages langagiers et espace. Les travaux pionniers de Weijnen (1946) et Rensink (1955) ont donné une impulsion à ce type de recherches qui, pendant cette première phase, se sont développés surtout aux Pays-Bas et au Japon. C'est seulement à partir des années 1980 que ces travaux trouvent un large écho en Amérique du Nord (v. Preston, 1996) et en Europe (v., entre autres, Kuiper, 1999 ; Iannàccaro \& Dell'Aquila, 2001 ; Boughton, 2006 ; Woehrling \& Boula de Mareüil, 2006).

Cet intérêt pour la perception de la variation régionale a été renforcé par l'essor des études se situant dans un champ nommé tantôt linguistique folk (angl. folk linguistics), tantôt linguistique profane ou populaire. Le développement de la linguistique folk est généralement attribué à Hoenigswald qui, dans un article publié en 1966, se prononce en faveur d'une prise en compte des savoirs spontanés. Selon Brekle, les objets de la linguistique populaire sont « tous les énoncés, les expressions "naturelles" - c'est-à-dire qui ne viennent pas des représentants de la linguistique comme discipline établie - qui désignent, ou se réfèrent à des phénomènes langagiers » (1984:48). Pour Achard-Bayle et Paveau, «le savoir spontané des acteurs sur le monde [...], qui se distingue du savoir savant ou scientifique [...] est constitué de connaissances empiriques, non susceptibles de vérification logique [...] et de croyances » (2008: 5 et suiv.).

Les deux perspectives sont généralement pensées en opposition : d'un côté la linguistique comme domaine scientifique ou savant, de l'autre la linguistique populaire qui reflète les opinions de sens commun, les évaluations spontanées des non-linguistes. De cette dichotomie découle la dichotomie entre "linguiste» et «locuteur profane » ou "nonlinguiste ». Si ce dernier peut être caractérisé comme un sujet «qui produit des discours métalinguistiques ne se fondant pas sur la linguistique scientifique » (Paveau, $2008: 148$ ), l'identification reste délicate. En effet, la catégorie des "non-linguistes » inclut un ensemble hétérogène d'individus, disposant de plus ou moins de connaissances sur les phénomènes langagiers. Selon le choix du sous-groupe de "non-linguistes », le résultat de la recherche amènera probablement le chercheur à des conclusions différentes.

Malgré cet aspect qui peut être considéré comme l'une des limites de cette approche, on peut affirmer, à l'instar de Paveau, que l'entrée perceptive dans la dimension sociale de la langue, en particulier à partir des méthodes de la dialectologie perceptuelle, « est à même de modifier le regard des linguistes sur leur objet et, partant, cet objet lui-même " (Paveau, 2008 : 154).

Dans une approche combinée qui met en perspective les données de production, de perception ainsi que les jugements affectifs et esthétiques associés à la variation diatopique, il ne s'agit pas de choisir entre données objectives et subjectives. $\mathrm{Au}$ contraire, toutes les informations possèdent une validité et sont susceptibles de nous éclairer, entre autres, sur les principes de catégorisation et de hiérarchisation de la variation langagière. Ainsi, les données évaluatives et perceptives peuvent aider à mieux comprendre les facteurs autres qu'intrasystémiques qui ont un impact sur la propagation ou l'abandon de faits de variation et, a fortiori, sur le changement linguistique ${ }^{3}$. 
31 Cette ouverture aux données «subjectives " ne permet cependant pas forcément de résoudre la question de l'association entre des pratiques langagières et un espace donné. Dans le cadre de la délimitation des variétés de français, Gadet souligne les limites d'une cartographie des faits langagiers variables, insistant sur le fait que «les usages non standards ou conservateurs [sont] rarement catégoriques (aucun locuteur un tant soit peu scolarisé n'en fait toujours usage) » et que « tout locuteur est susceptible d'alterner selon les situations. C'est ici encore la question des données, du fait de l'omniprésence de la variabilité, même dans le vernaculaire regardé par les variationnistes comme le lieu de la cohérence » $(2017: 85)$.

Or, derrière cette cohérence ou homogénéité, figurant sous l'étiquette parler de $X$, français de $X$ se cache une hétérogénéité qui peut facilement être démontrée si l'on s'intéresse de près aux usages variables de locuteurs «représentatifs » de l'espace en question (v. Falkert, 2010). De ce fait, un regard critique sur les "preuves", notamment en géolinguistique, s'impose, puisque « dès lors que l'on élargit et diversifie les données, le paysage variationnel se nuance et se complexifie. Et la plupart de ces données échappent autant à l'intuition du linguiste qu'à beaucoup de corpus, surtout pour l'ordinaire et le spontané » (Gadet, $2017: 84)$.

\section{Au-delà du clivage qualité-quantité}

Produire des connaissances dans des conditions instables, dans un monde qui change continuellement, représente un défi considérable pour le chercheur. Les exigences d'objectivité et de théorisation (ou de "modélisation») sont étroitement liées à la présence de statistiques. Si la quantification crée un langage commun (par les chiffres), on peut se demander dans quelle mesure elle intervient dans la construction de la preuve.

Les enquêtes par questionnaire et les corpus oraux invitent à une exploitation quantitative des données. Or, si l'on veut « produire des images du monde prenant appui sur des nombres » (Desrosières \& Didier, 2014), il est légitime de s'interroger sur le poids que ces chiffres peuvent et doivent prendre dans une argumentation scientifique. Malheureusement, les choix du chercheur ne sont pas toujours clairement explicités. Pour ce qui est des enquêtes par questionnaire, par exemple, on peut s'interroger sur les seuils définis par le chercheur : à partir de quel pourcentage de réponses convergentes peut-on considérer une réponse comme une "preuve » ? Concernant les corpus, il serait légitime de se demander à partir de combien d'attestations on peut conclure à la diffusion d'un fait langagier ? On peut regretter que les choix effectués par le chercheur ne font pas systématiquement l'objet d'explications dans les travaux de géolinguistique. Certaines études sur la variation du français se contentent volontairement d'étiquettes comme "souvent» et "partiellement» pour décrire des usages et tirer des conclusions sur l'extension des traits linguistiques (v. Neumann-Holzschuh \& Wiesmath, 2006). Faute de corpus comparables et eu égard à l'hétérogénéité des sources sur lesquelles s'appuient certaines recherches, il est en effet difficile de fournir des indications précises, chiffrées. Cela dit, ce fait ne dispense pas le chercheur de prendre position par rapport au niveau de granularité ou de «simplification » de la réalité linguistique complexe qu'il retient pour son argumentation.

35 Autrement dit, une prise de conscience des critères appliquée et une réflexion sur l'impact de ce choix permettraient de mieux rendre compte du poids qui peut être 
attribué aux données-preuves. Par ailleurs, dans une démarche de réduire la complexité de la réalité (ou, pour le dire avec les mots de Becker [2007: 96] : « to make a little out of a lot »), un regard sur le degré homogénéité des données (et des informateurs) donne une meilleure image de l'importance des preuves avancées. Dans des travaux, notamment dans ceux qui prétendent à une théorisation ou une modélisation, le calcul de l'écart-type et de la distribution permettrait de nuancer les résultats obtenus et d'éviter le risque d'une déformation de la réalité. Comme nous l'avons vu dans le chapitre précédent, derrière l'homogénéité apparente d'un parler, d'une variété, peut se cacher une hétérogénéité qu'une étude détaillée des profils langagiers individuels permet de rendre visible (v. Falkert, 2010). C'est dans cette optique que l'articulation entre le qualitatif et le quantitatif prend tout son sens.

\section{Quelle place pour les données partielles et les cas isolés?}

36 L'enquête de terrain réserve souvent des surprises, positives ou négatives. Pour le chercheur, cela implique la production de connaissances inattendues, parfois contradictoires, qui empêchent de tirer des conclusions définitives. Les données partielles, incomplètes, représentent un vrai casse-tête pour le scientifique. Parfois, le problème ne peut être résolu en effectuant une enquête supplémentaire sur le terrain. Le chercheur est alors obligé de " faire avec » et décider d'intégrer ou non ces données dans l'analyse. Pour ne prendre qu'un exemple, il suffit de regarder le traitement des « cartes mentales» en dialectologie perceptuelle. Afin d'obtenir des informations sur la représentation de la variation diatopique et le découpage de l'espace dialectal par les locuteurs naïs, l'une des méthodes de recueil de données consiste à demander aux informateurs de dessiner des lignes ou des cercles délimitant des aires dialectales imaginées sur des fonds de carte. Or, cette tâche exige des connaissances en géographie que tous les informateurs n'ont pas. La difficulté à exploiter les données provenant des cartes perceptuelles dessinées par les natifs et comportant des informations soit incomplètes, soit inexploitables puisque l'informateur n'arrive pas à se repérer sur une carte, a été soulignée par Falkert (2012) et plus récemment par Gally (2017) :

Nous avons été confrontée à des difficultés de classement et de traitement des cartes mentales. En présence des erreurs d'identification et de projection géographique [...], nous avons décidé de ne pas prendre en compte les croquis de ces participants dans les analyses globales. (Gally, 2017 : 292)

On revient alors à la question de la « représentativité » de ce type de données partielles. À l'ère de la "modélisation », cela pose évidemment problème puisque les résultats ne se prêtent pas à une théorisation, ces données partielles étant basées uniquement sur les informations d'une partie des enquêtés, à savoir ceux qui ont réussi à se repérer sur le fond de carte. Il appartient alors au chercheur, en s'appuyant sur sa connaissance du terrain d'enquête, d'évaluer le poids en tant que « preuves » qu'il peut raisonnablement attribuer à ces données et de l'expliciter. C'est également vrai pour les cas isolés qui sont souvent mis sur le compte de la variation aléatoire. Becker (2016) avance que les cas isolés peuvent annoncer des tendances, des changements à venir et qu'ils ont, de ce fait, toute leur place dans l'argumentation scientifique. On revient ainsi au pouvoir heuristique des données, qui ne servent pas qu'à confirmer ou infirmer des hypothèses, mais qui ouvrent des perspectives pour de nouvelles problématiques de recherche. 


\section{Le recueil de données géolinguistiques à l'ère du numérique}

D'un point de vue méthodologique, on observe, à l'heure actuelle, la coexistence de démarches «traditionnelles» (enquêtes de terrain, entretiens) et de méthodes innovantes qui tirent profit des possibilités ouvertes par le Web 2.0. Si le recueil de données par Internet offre des perspectives nouvelles, il présente aussi quelques inconvénients. La technique du crowdsourcing (ou production participative) reçoit, depuis quelques années, une attention grandissante dont témoigne, entre autres, une journée d'étude intitulée "Variation linguistique et crowdsourcing: étudier la variation au $\mathrm{xxI}^{\mathrm{e}}$ siècle ", organisée à l'université de Strasbourg en octobre 2015. Dans le domaine des sciences du langage, on remarque un essor des recherches s'appuyant sur la production participative (v. Eskenazi et al., 2013). En vue de documenter sous un nouveau jour la variation régionale, le crowdsourcing est utilisé dans plusieurs projets exploitant cette méthode à travers des plateformes dédiées, notamment Verba Alpina ${ }^{4}$, Français de nos régions ${ }^{5}$ et Donnez votre français à la science ${ }^{6}$. Dans le cadre d'une enquête sur la variation diatopique du français dont le but était d'évaluer la vitalité de certaines tournures régionales, ainsi que leur extension géographique, Avanzi et al. (2016) avaient recueilli les réponses de plus de 12000 participants. Pour ce qui est de la partie concernant le lexique, les participants devaient choisir, à l'intérieur d'une liste proposée, l'item qu'ils utilisent préférentiellement pour désigner l'objet en question. La modalité « autre » pour indiquer un terme qui ne figurait pas dans la liste était également proposée. Les participants devaient ensuite renseigner sur une échelle de Likert modifiée allant de 0 (= jamais) à 10 (= très souvent) la fréquence à laquelle ils utilisent telle ou telle expression (v. Avanzi et al., 2016).

Cette méthode permet, en effet, de recueillir, dans un laps de temps réduit, une grande quantité de données. Elle comporte cependant des inconvénients qui relèvent de trois niveaux et qui obligent à s'interroger sur le statut de "preuves » des données ainsi recueillies. Premièrement, l'absence de «contrôle» sur les informateurs fait que l'enquêteur ne peut réellement savoir qui a répondu à l'enquête. L'indication d'un profil sociobiographique que les participants doivent compléter avant ou après l'enquête ne dit rien sur la véracité des informations données. Le temps de réponse n'étant le plus souvent pas limité, le caractère "spontané" des réponses n'est pas garanti. Deuxièmement, la répartition inégale des participants selon les régions peut rendre l'exploitation des données difficile, comme l'ont constaté Boula de Mareüil et al. dans le cadre d'une recherche sur des variantes de prononciation en français :

Pour tracer plus précisément des isoglosses, le besoin va se faire sentir de récolter davantage de données dans les zones de transition entre domaines d'oïl et d'oc. Malheureusement, celles-ci correspondent souvent aux régions les moins peuplées de la France, des régions rurales qui se montrent parfois rétives au maillage du territoire - ceci constitue une limite de la méthodologie à base de crowdsourcing. $(2016: 126)$

Enfin, les données ainsi recueillies sont basées sur des comportements déclarés qui mériteraient d'être confrontés aux usages réels :

Nous sommes bien conscients qu'une telle consigne [indiquer la tournure/ expression qu'ils utiliseraient spontanément au cours d'une conversation familière pour verbaliser une situation décrite sur une image, A.F.] ne constitue pas une 
garantie absolue d'obtenir des résultats reflétant les pratiques réelles, en particulier pour des locuteurs qui pourraient être en forte insécurité linguistique.

(Avanzi et al., $2016: 3$ )

41 Malgré ces inconvénients, il est évident que l'utilisation du crowdsourcing constitue un enrichissement pour la recherche sur la variation diatopique et que les enquêtes impliquant davantage les locuteurs naïfs fournissent des renseignements précieux, susceptibles de faire émerger de nouvelles problématiques de recherche en géolinguistique.

\section{Conclusion}

Dans un souci d'enrichir la réflexion épistémologique et méthodologique, nous venons d'avancer quelques remarques concernant l'évaluation critique des données transformées en «preuves» dans le cadre de l'argumentation scientifique. Ces observations ne se veulent pas une critique des travaux réalisés dans le domaine de la géolinguistique, mais un plaidoyer pour une meilleure prise en compte de ces aspects dans la formation de nos étudiants en master et en doctorat. À notre avis, l'enjeu consiste à sensibiliser les jeunes chercheurs à la prudence qui s'impose dans le cadre d'une argumentation scientifique, surtout quand on travaille avec des données mises à disposition par d'autres chercheurs. Se demander dans quelles conditions elles ont été recueillies, par qui, dans quel but, et adopter une approche où l'on compare ce qui est réellement comparable sont autant d'impératifs qui contribuent à la crédibilité des résultats.

Peut-on ainsi affirmer, à l'instar de Gadet, que «tout est question de données et de l'endroit où on les cherche » $(2017: 81)$ ? Probablement, mais il faudrait sans doute ajouter « et de la capacité du chercheur à évaluer le potentiel des données à constituer des "preuves" dans l'argumentation scientifique». Nous souhaitons ainsi encourager les jeunes chercheurs en géolinguistique à développer « la volonté de ne pas croire, du moins en l'absence d'indice solide de vraisemblance» (Peneff, 2009:165), de se méfier des raccourcis, et de procéder, à chaque étape de la recherche, à une évaluation critique des données en tenant compte du contexte dans lequel elles ont été recueillies. Les protocoles standardisés et guides de bonnes pratiques qui constituent les références incontournables des cours de méthodologie de la recherche ne devraient pas faire oublier la nécessité de s'interroger sur la validité des connexions logiques établies entre données, preuves et idées et de se positionner par rapport aux critères qui permettent d'attribuer la valeur de " preuve » à ces mêmes données. En résumé, ce n'est pas seulement en prenant du recul, mais surtout en multipliant les distances et les angles que le chercheur, comme le photographe, a les meilleures chances de décrypter les situations et les comportements qu'il s'efforce de décrire. 


\section{BIBLIOGRAPHIE}

Achard-Bayle Guy \& Paveau Marie-Anne, 2008, « La linguistique "hors du temple" », Pratiques, n 139-140, p. 3-16.

Avanzi Mathieu, Barbet Cécile, Glikman Julie \& Peuvergne Julie, 2016, « Présentation d'une enquête pour l'étude des régionalismes du français ", dans F. Neveu, G. Bergounioux, M.-H. Côté, J.-M. Fournier, L. Hriba \& S. Prévost (éds), SHS Web of Conferences (actes du $5^{\mathrm{e}}$ Congrès mondial de linguistique française), vol. 27, article $\mathrm{n}^{\circ}$ 3001, p. 1-14. <https://doi.org/10.1051/ shsconf/20162703001>.

BAUde Olivier \& BLANCHE-BENVEnISTE Claire (éds), 2006, Corpus oraux : guide des bonnes pratiques, Orléans, Presses universitaires d'Orléans.

BEAUD Stéphane \& WEBER Florence, 2017, Guide de l'enquête de terrain : produire et analyser des données ethnographiques, Paris, La Découverte.

BECKER Howard S., 1963, Outsiders: Studies in the Sociology of Deviance, New York, Free Press.

BECKER Howard S., 1982, Art Worlds, Berkeley, University of California Press.

BECKER Howard S., 2007, Telling about Society, Chicago, The University of Chicago Press.

BECKER Howard S., 2014, What about Mozart? What about Murder? Reasoning from Cases, Chicago, The University of Chicago Press.

BECKER Howard S., 2016, La bonne focale : de l'utilité des cas particuliers en sciences sociales, Paris, La Découverte.

BECKER Howard S., 2017, Evidence, Chicago, The University of Chicago Press.

Boughton Zoe, 2006, « When Perception Isn't Reality: Accent Identification and Perceptual Dialectology in French », Journal of French Language Studies, n 16, p. 277-304.

Boula de MareüIl Philippe, Goldman Jean-Philippe, RILliard Albert, ScherRer Yves \& VerniER Frédéric, 2016, « Cartopho : un site web de cartographie de variantes de prononciation en français ", dans Actes des 31 es Journées d'études sur la parole (JEP-TALN-RECITAL), vol. 1, p. 119-127. Disponible sur <https://jep-taln2016.limsi.fr/actes/Actes\%20JTR-2016/Papers/J28.pdf>.

BOWERN Claire, 2008, Linguistic Fieldwork: A Practical Guide, New York, Palgrave Macmillan.

BREKLE Herbert E., 1984, « La "linguistique populaire” : est-elle un objet possible d'une historiographie de la linguistique ? ", Histoire Épistémologie Langage, première série, nº 5, p. 47-59.

CAPPEAU Paul \& GADET Françoise, 2007, « L'exploitation sociolinguistique des grands corpus.

Maitre-mot et pierre philosophale ", Revue française de linguistique appliquée, vol. 12, n 1 , p. $99-110$.

CukoR-Avila Patricia \& BAILey Guy, 1995, « An Approach to Sociolinguistic Fieldwork: A Site Study of Rural AAVE in a Texas Community », English World-Wide, nº 16, p. 159-193.

DESROSIÈRES Alain, 2010, La politique des grands nombres : histoire de la raison statistique, Paris, La Découverte. 
DESROSIÈRES Alain \& DIDIER Emmanuel, 2014, Prouver et gouverner : une analyse politique des statistiques publiques, Paris, La Découverte.

DETEY Sylvain, 2017, « La variation dans l'enseignement du français parlé en FLE : des recherches linguistiques sur la francophonie aux questionnements didactiques sur l'authenticité ", dans A.C. Jeng, B. Montoneri \& M.-J. Maitre (éds), Échanges culturels aujourd'hui : langue et littérature, New Taipei City, Tamkang University Press, p. 93-114.

Eskenazi Maxine, Levow Gina-Anne, Meng Helen, Parent Gabriel \& Suendermann David, 2013, Crowdsourcing for Speech Processing: Applications to Data Collection, Transcription, and Assessment, Chichester, John Wiley \& Sons Ltd.

FALKERT Anika, 2010, Le français acadien des Îles-de-la-Madeleine. Étude de la variation phonétique, Paris, L'Harmattan.

GADET Françoise, 2017, « Variatio delectat : variation et dialinguistique », Langage et société, $\mathrm{n}^{\circ}$ 160-161, p. 75-91. <https://doi.org/10.3917/ls.160.0075>.

GADET Françoise, Ludwig Ralph, Mondada Lorenza, PfäNder Stefan \& SIMON Anne-Catherine, 2012, « Un grand corpus de français parlé : le CIEL-F. Choix épistémologiques et réalisations empiriques ", Revue française de linguistique appliquée, vol. 17, nº 1, p. 39-54.

GADET Françoise \& WACHS Sandrine, 2015, « Comparer des données de corpus : évidence, illusion ou construction ? ", Langage et société, $\mathrm{n}^{\circ} 154$, p. 33-49.

GALLY Silvia, 2017, Identités linguistiques perçues : quelques localités des «vallées vaudoises » du Piémont occidental (Italie), thèse de doctorat, Université Grenoble Alpes.

GoEBL Hans, 1982, Dialektometrie: Prinzipien und Methoden des Einsatzes der Numerischen Taxonomie im Bereich der Dialektgeographie, Vienne, Österreichische Akademie der Wissenschaften.

GUITER Henri, 1973, « Atlas et frontières linguistiques », dans G. Straka et P. Gardette (éds), Les dialectes romans de France à la lumière des atlas régionaux. Actes du colloque de Strasbourg (24-28 mai 1971), Paris, Éditions du CNRS, p. 61-109.

HEERINGA Wilbert, 2004, Measuring Dialect Pronunciation Differences Using Levenshtein Distance, Thèse de doctorat, Rijksuniversiteit Groningen.

HoEnigswALD Henry, 1966, « A Proposal for the Study of Folk Linguistics », dans W. Bright (éd.), Sociolinguistics: Proceedings of the UCLA Sociolinguistic Conference 1964, La Hague, Mouton, p. 16-26.

IANNÀcCARo Gabriele \& Dell'AQUila Vittorio, 2001, « Mapping Languages from Inside: Notes on Perceptual Dialectology », Social \& Cultural Geography, vol. 2, n 3, p. 265-280.

KUIPER Lawrence, 1999, « Variation and the Norm. Parisian Perceptions of Regional French », dans D. Preston (éd.), Handbook of Perceptual Dialectology, Amsterdam, Benjamins, p. 241-262.

LABOV William (2001 [1975]), « Qu'est-ce qu'un fait linguistique? » [traduction de « What Is a Linguistic Fact? »], Marges linguistiques, 1, p. 25-68. Disponible sur <www.revue-texto.net/ Parutions/Marges/Marges_sommaire.html>.

MATTHEIER Klaus, Gilles Peter, SCHARLOTH Joachim \& Ziegler Evelyn (éds), 2010, Variatio delectat: empirische Evidenzen und theoretische Passungen sprachlicher Variation, Francfort-sur-le-Main, New York, Peter Lang.

Neumann-Holzschun Ingrid \& Wiesmath Raphaële, 2006, « Les parlers acadiens : un continuum discontinu », Revue canadienne de linguistique appliquée, vol. 9, nº 2, p. 233-249. 
PAVEAU Marie-Anne, 2008, «Le parler des classes dominantes, objet linguistiquement incorrect? Dialectologie perceptive et linguistique populaire », Études de linguistique appliquée, $\mathrm{n}^{\circ} 150$, p. 137-156.

PENEFF Jean, 1995, « Mesure et contrôle des observations dans le travail de terrain. L'exemple des professions de service ", Sociétés contemporaines, $\mathrm{n}^{\circ} 21$, p. 119-138. <https://doi.org/10.3406/ socco.1995.1423>.

PENEFF Jean, 2009, Le goût de l'observation : comprendre et pratiquer l'observation participante en sciences sociales, Paris, La Découverte.

PRESTON Dennis, 1996, « “Whaddayaknow': The Modes of Folk Linguistic Awareness », Language Awareness, vol. 5, $\mathrm{n}^{\circ}$ 1, p. 40-74.

RENSINK Wim, 1955, « Dialectindeling naar opgaven van medewerkers », Mededelingen Centrale Commissie tot onderzoek van het Nederlands Volkseigen, $\mathrm{n}^{\circ}$ 7, p. 20-23. [Traduction de «Informant Classification of Dialects ", dans D. Preston (éd.), Handbook of Perceptual Dialectology, Amsterdam, John Benjamins, 1999, p. 3-7.]

SCHEER Tobias, 2013, « The Corpus: A Tool among Others », Corela, hors-série $\mathrm{n}^{\circ}$ 13, sans pagination.

SÉGUY Jean, 1971, « La relation entre la distance spatiale ct la distance lexicale », Revue de linguistique romane, $\mathrm{n}^{\circ} 35$, p. 335-357.

SÉGUY Jean, 1973, « La fonction minimale du dialecte », dans G. Straka et P. Gardette (éds), Les dialectes romans de France à la lumière des atlas régionaux. Actes du colloque de Strasbourg (24-28 mai 1971), Paris, Éditions du CNRS, p. 27-42.

THомаs Erik R., 2002, « Sociophonetic Applications of Speech Perception Experiments », American Speech, $\mathrm{n}^{\circ} 2$, p. 115-147.

VAUX Bert \& COOPER Justin, 2005, Introduction to Linguistic Field Methods (2 éd.), Munich, LINCOM Europe.

VIAUT Alain, 2004, « La frontière linguistique de la ligne à l'espace : éléments pour une schématisation ", Glottopol, nº 4, p. 6-22.

WEIJNEN Antonius, 1946, « De grenzen tussen de Oost-Noordbrabantse dialecten onderling », dans A. Weijnen, J. M. Renders \& J. Van Ginneken (éds), Oost-Noordbrabantse dialectproblemen. Bijdragen en Mededelingen der Dialectencommisie van de Koninklije Nederlandse Akademie van Wetenschappen te Amsterdam, vol. 8, Amsterdam, Noord-Hollandsche U. M., p. 1-15.

Woehrling Cécile \& Boula De MareüIL Philippe, 2006, « Identification of Regional Accents in French: Perception and Categorization », dans Proceedings of the 9th International Conference on Spoken Language Processing, Pittsburgh, p. 1511-1514.

\section{NOTES}

1. ESLO : Enquête sociolinguistique à Orléans ; CID : Corpus of Interactional Data ; CLAPI : Corpus de langues parlées en interaction; PFC: Phonologie du français contemporain; VALIBEL: Variétés linguistiques $\mathrm{du}$ français en Belgique; VARILING: traitement des Variations linguistiques dans les corpus.

2. <www.ciel-f.org>. 
3. Voir aussi la discussion concernant la prise en compte de données "subjectives » dans Mattheier et al. (2010).

4. <www.verba-alpina.gwi.uni-muenchen.de>.

5. <https://francaisdenosregions.com>.

6. <http://donnezvotrefrancais.fr>.

\section{RÉSUMÉS}

Dans cet article, nous nous interrogerons sur les éléments qui fondent la validité des démonstrations en géolinguistique et sur les questions méthodologiques qui y sont liées. En nous appuyant sur les arguments avancés par le sociologue américain Howard Becker dans son ouvrage intitulé Evidence, nous tenterons de déceler les enjeux relatifs à la « construction » de la "preuve» dans les recherches sur la variation diatopique et nous soulignerons leur pertinence dans le cadre de la formation de jeunes chercheurs.

In this article, we will examine the elements that underpin the validity of geolinguistic demonstrations and discuss related methodological issues. Relying on the arguments put forward by the American sociologist Howard Becker in his book Evidence, we will attempt to identify issues related to the "construction" of "proof" in studies in the field of dialect geography, and we will highlight their relevance for research training programs aimed at graduate and postgraduate students.

\section{INDEX}

Keywords : Evidence, geolinguistics, methodology, data

Mots-clés : preuve, géolinguistique, méthodologie, données

\section{AUTEUR}

\section{ANIKA FALKERT}

Université d'Avignon, Laboratoire ICTT (EA 4277)

anika.falkert@univ-avignon.fr 